\title{
Dynamic stereo displays for research on the recovery of three-dimensional structure
}

\author{
MYRON L. BRAUNSTEIN \\ University of California, Irvine, California
}

\begin{abstract}
Research issues that can be addressed by combining stereoscopic and kinetic depth displays include cue conflict and recalibration, mutual constraints, specialization of cues, and differential effects of disparity and kinetic depth on other perceptions, such as size constancy. Methods of producing dynamic stereoscopic displays are reviewed, especially displays combining stereoscopic with orthographic projections of rotation in depth. A sample personal computer program in Pascal is provided.
\end{abstract}

Two of the most important contributions of computer technology to vision research have been in the areas of stereoscopic depth perception (Julesz, 1960) and kinetic depth perception (Green, 1961). There are important reasons for bringing together these two areas of research, and during the past several years a number of investigators have made use of current computer technology to do so. This paper considers research issues that have been or can be addressed by combining binocular disparity and kinetic depth in dynamic stereoscopic displays. The first part reviews five major research issues, some of which overlap: cue conflict and recalibration, mutual constraints, specialization of cues, dynamic versus static disparity, and implications of disparity and kinetic depth for other perceptual judgments. The second part considers implementation. Calculations required for dynamic stereoscopic displays are discussed, including displays in which the stereo pairs are constructed from frames in the motion sequence. Methods of viewing these displays are reviewed, and a sample program in Pascal is provided in an Appendix.

\section{RESEARCH ISSUES}

\section{Cue Conflict and Recalibration}

In studying alternative sources of information for depth perception, one can usually put two or more sources in conflict and determine which is dominant. This approach by itself may not provide especially interesting data, as it is usually possible to increase the magnitude of some quantity relevant to one source and decrease the magnitude of some quantity relevant to a second source until the first source appears dominant. There are, however,

Preparation of this paper received support from National Eye Institute Grant EY 04553 to the author and from a contract to D. Hoffman from the Office of Naval Research, Psychological Sciences Division, Engineering Psychology Group. I am grateful to George J. Andersen for valuable discussions of this work and to Lionel Shapiro and James Tittle for comments and assistance in the preparation of the manuscript. The sample program is based on one written by James Tittle. The author's address is: Myron L. Braunstein, Cognitive Sciences Group, School of Social Sciences, University of California, Irvine, CA 92717. questions of broader interest that can be asked about the effects of inconsistent information from alternative sources. One important question is whether prolonged exposure to conflicting information will cause a recalibration of the relationship between the information from one or both sources and perceptual judgments. For example, if an observer perceives a particular amount of depth in a three-dimensional pattern on the basis of disparity, will exposure to conflicting kinetic depth information over a period of time alter this relationship between disparity and perceived depth? This question was addressed by Wallach, Moore, and Davidson (1963) and by Epstein (1968). In Epstein's experiment, subjects first judged depth between a pair of points viewed binocularly. Next, they observed a rotating wire octahedron, with stereoscopic depth exaggerated through the use of a mirror telestereoscope. When the subject was given the dot judgment task again, the relationship between judged depth and disparity was found to be altered in a direction consistent with the kinetic depth information: Less depth was perceived for a given amount of disparity than before exposure to the conflicting-information trials. An important finding of this research was that kinetic depth recalibrated stereoscopic depth, but recalibration in the reverse direction did not occur (Wallach et al., 1963). This result anticipated recent analyses of the relationships between kinetic depth and disparity by Braunstein (in press), Prazdny (1986), and Richards (1985), which suggest that kinetic depth is a more direct and perhaps a more reliable source of information about object-centered depth (shape or spatial layout) than is disparity. These analyses are discussed below.

The Wallach et al. (1963) and Epstein (1968) studies provided subjects with inconsistent information about amount of depth within a three-dimensional configuration. It is also possible to consider conflicting information for depth order, defined as the order of feature points in depth from the observer (also called signed depth or disposition). Disparity, for most stimuli, provides information about relative distances between the observer and feature points in the environment. Depth order is usually unambiguous. On the other hand, kinetic depth, when based on an ortho- 
graphic projection, provides information about object shape but leaves depth order ambiguous. Depth order can be added from other information, such as occlusion (Andersen \& Braunstein, 1983; Braunstein, Andersen, \& Riefer, 1982) or relative brightness (Schwartz \& Sperling, 1983), or by changing to a polar projection (Braunstein, 1966), in effect adding perspective to the display.

Braunstein, Andersen, Rouse, and Tittle (1986) addressed the question of whether these supplementary sources of information for depth order remained effective even when unambiguous information about depth order was available from binocular disparity. The stimuli in one experiment were stereo pairs representing pentagonal texture elements on the surface of a sphere rotating about a vertical axis (see Figure 1). Two types of occlusion were simulated: edge occlusion, in which texture elements were occluded as they rounded the edge of an opaque sphere, and element occlusion, in which nearer texture elements on a transparent sphere covered and uncovered more distant elements. The depth order indicated by occlusion either corresponded to or conflicted with the depth order indicated by disparity. Direction-of-rotation judgments were used as a measure of perceived depth order. We found that these judgments were made in accordance with the stereoscopic information on $94 \%$ of the element occlusion trials and on $91 \%$ of the edge occlusion trials when disparity and occlusion information were in correspondence. When the two sources were in conflict, the proportion of judgments made in accordance with the stereoscopic information dropped to $81 \%$ for the element occlusion displays and to $36 \%$ for the edge occlusion displays.

In a second experiment, Braunstein et al. (1986) considered information for depth order from disparity and from velocity gradients. The stimuli were dots uniformly distributed in the image plane, translating horizontally. The translation speed of a dot in the image plane was proportional to its vertical distance from the center of the image plane, simulating a dihedral angle with the apex pointed toward or away from the subject. The angle orientation (relative to the subject) indicated by disparity was either the same as or opposite to that indicated by the velocity gradient. Conflicting velocity gradient information significantly reduced the proportion of angle-orientation judgments ("center-near" vs. "center-far") made in accordance with the stereoscopic information. The impact of the conflicting monocular information on these judgments was greater when the velocity gradient indicated a center-near (convex) orientation and was greater at the higher of the two translation speeds studied. Overall, these two experiments demonstrated that sources of information used to resolve depth order ambiguities in monocular displays remain effective even when unambiguous information for depth order is available from stereoscopic information.

The monocular information for three-dimensional structure in the displays used in these two experiments is representative of two types of dynamic monocular infor- mation from which three-dimensional structure may be recovered. In discussions of research relating stereoscopic depth with depth from motion, it is useful to distinguish between these two types of monocular information. In both experiments, a simulated object was moving rigidly in three dimensions, so that the three-dimensional velocities of all feature points on the object were the same. The velocity gradient display in the second experiment was a polar projection in which the projected velocities of the feature points varied inversely with their distances from the projection point. This display simulates the velocity variations of projected points on the retina that correspond to texture elements moving at the same velocities, relative to the eye, in three-dimensional space. (The simulation is approximate, as it treats the retina as a plane.) This type of gradient would be produced by motion of the observer orthogonal to the line of sight, for example, by the observer's looking $90^{\circ}$ to the side while moving forward. It is appropriately described as motion parallax.

It is sometimes argued that motion parallax produces only small variations in projected velocity, but this is, of course, a function of the relative viewing distances involved. The projected velocity of a point is inversely proportional to its distance from the projection point. Consider, for example, a surface that extended $1 \mathrm{~m}$ in depth with the closest point $1.5 \mathrm{~m}$ from the eye. If the surface translates perpendicularly to the line of sight, the projected velocity of the nearest point on the surface will be approximately double the projected velocity of the most distant point. Motion parallax is usually associated with translation, although differences between near and far points at the same orientation in the polar projection of a rotation can also be regarded as motion parallax effects.

The rotating sphere display in the first experiment was an example of displays to which Wallach and O'Connell (1953) have applied the term kinetic depth effect. The kinetic depth effect is based on changes in the orientation of the motion path of a feature point relative to the image plane. These changes, which occur during rotation about any axis other than the line of sight, alter the length of the projected motion path and thus alter the projected velocities of points moving at the same three-dimensional velocities. These changes in projected velocity occur in orthographic projections, as well as in polar projections, and are not due to changes in distance between the points and the observer. More recently, the expression structure from motion has become widely used to describe this type of display (Ullman, 1979). This expression is not appropriate, however, for distinguishing between the recovery of three-dimensional structure from orthographic projections of rotation and the recovery of structure from motion parallax. Recovery of structure from motion parallax displays-polar projections of horizontal translations-has been clearly demonstrated by Rogers and Graham (1979). Although "kinetic depth effect" is also not an ideal expression (as it could refer to any type of motion that implies depth), it is convenient to restrict this expression to the recovery of structure from orthographic 
projections of rotations, to distinguish this type of display from motion parallax displays. (Dividing optic flow into motion parallax and kinetic depth is a simplification. For a comprehensive discussion of the information available for shape perception in optic flow, see Koenderink, 1986.)

For some stimuli, motion parallax effects are similar to kinetic depth effects, when considered locally in space and time. Consider, for example, the velocity gradient in a polar projection of a slanted surface translating horizontally. This gradient occurs in the image plane because the projected velocities of points moving at equal velocities in three-dimensional space are a function of their distance from the observer. If the surface were rotating about a vertical axis rather than translating along a horizontal axis, the projected velocities would vary with the distances of the points from the axis of rotation in the depth dimension. (This is true for a limited range of horizontal distances from the axis and a limited angle of rotation.) Either a motion parallax analysis or a kinetic depth analysis might recover a slanted surface, but a kinetic depth analysis with the projection treated as orthographic would leave the depth order (the sign of the slant in this example) ambiguous. Hoffman (personal communication, February 1984) suggested that observers viewing a velocity gradient produced by translating a surface horizontally initially apply an orthographic analysis locally (in space and time) to recover the structure. This suggestion is supported by two findings with monocular displays (Braunstein \& Andersen, 1981). First, subjects recovered the shape of a dihedral angle in about $3 \mathrm{sec}$ of viewing but did not become accurate in judging the orientation of the angle unless the viewing time was extended to about $10 \mathrm{sec}$. Second, when subjects were asked to rate perceived rotation in these displays, rotation judgments were highly correlated with the steepness of the velocity gradient.

This perceived rotation did not occur during monocular viewing when the translation of the dots was linked to head movements by the observer (Rogers \& Graham, 1979). This would seem to indicate that a motion parallax analysis of a velocity gradient requires active head movement. However, Rogers and Graham (1979) and Rogers and Collett (1986) obtained results similar to the head movement results by moving the monitor, with the observer's head stationary. We have found, in research in progress, that motion parallax effects can be obtained on a stationary monitor by placing the display in a "window" within the monitor and moving the window back and forth horizontally across the screen, essentially simulating monitor motion. These observations suggest that it is relative motion between the contours of the display and the observer that is important in producing a motion parallax effect.

It is possible that the recovery of three-dimensional structure from motion parallax makes use of visual processes that are related to the perception of observer motion. When dot motion is linked to head movements, this relationship is obvious. When the monitor is moved relative to the eye, there may still be a tendency to apply constraints appropriate to the head-movement situation (e.g., that surfaces in the environment are moving rigidly relative to the observer). This relationship between recovery of structure from motion parallax and the application of constraints appropriate to observer motion is speculative. Our understanding of motion parallax should benefit from research on how the visual system segregates regions of optic flow into those attributable to observer motion and those attributable to object motion (Andersen, 1986).

\section{Mutual Constraints}

There are an infinite number of three-dimensional interpretations for any two-dimensional image or any pair of two-dimensional images, and computational analyses of vision have attempted to determine environmental constraints that might be exploited by an observer to reduce this infinity of possibilities to a unique solution. Richards (1985) has argued that stereo and motion, considered separately, each fails to specify uniquely the structure and disposition of a three-dimensional object. Disparity may provide an indication of structure, but the depth scale may be distorted. An orthographic projection of a rotating figure, with sufficient numbers of points and views, can provide the structure of the object, but the depth order or disposition relative to the observer is ambiguous. Richards shows that a combination of stereo and motion can provide both undistorted information for structure and unambiguous depth order. Waxman and Duncan (1985) have also presented an analysis of how stereo and motion information may be combined, relating their "stereomotion fusion module" to psychophysical and neurophysiological studies with changing disparity stimuli by Regan and Beverley (1979). Empirical research on the mutually constraining effects of stereo and motion is just beginning to appear (Lappin \& Love, 1986).

\section{Specialization of Cues}

Stereo and kinetic depth appear to provide qualitatively different types of information about the three-dimensional environment. This concept is closely related to the mutual constraint analysis discussed in the previous section, but also applies when these sources of information are considered in isolation. Information from disparity appears to fit the Marr (1982) paradigm, first providing a viewercentered representation (the $21 / 2$-dimensional sketch) from: which an object-centered representation (the threedimensional model) is derived. A kinetic depth analysis, on the other hand, does not seem to require a viewercentered stage, as three-dimensional coordinates in an arbitrary object-centered coordinate system can be recovered directly from motion in the two-dimensional image. It may be that stereo is specialized for viewer-centered depth and kinetic depth information is specialized for object-centered depth and kinetic depth information is specialized for object-centered depth (Braunstein, in press). Prazdny (1986) has made a similar distinction, describing the binocular correlation mechanism 
as a depth-measurement device and the structure-frommotion mechanism as a layout-sensing device. This distinction may not apply to all aspects of disparity, however. Current research by Rogers (1986) suggests that the second derivative of disparity, and also of motion parallax, provides information about curvature. This information may feed directly into an object-centered representation. It may be generally true that higher derivatives of viewercentered information are direct sources of object-centered information.

\section{Dynamic Disparity}

Regan and Beverley (1979) reported greater sensitivity to a stereo view simulating a square moving in depth, combining changing size and changing disparity, than to either change alone. We have found that some subjects who were unable to respond to static disparities (Braunstein et al., 1986) could respond accurately to depth order indicated by changing disparities. The stimuli were similar to those shown in Figure 1, except that the hidden lines were not removed. There was, thus, no monocular information for depth order, and it could only be recovered from stereoscopic information. We showed these stimuli to 17 subjects who had failed a static stereo test (Random Dot E) given to 235 students. Six subjects, who were also unable to detect even the largest disparity ( $400 \mathrm{sec}$ of arc) on the Random Dot Circles test, responded in accordance with disparity to $90 \%$ or more of the changing-disparity stimuli. Stereoscopic versions of orthographic projections of objects rotating in depth may be useful to supplement static tests of stereoacuity in clinical settings. (See also Luria \& Weissman, 1968).
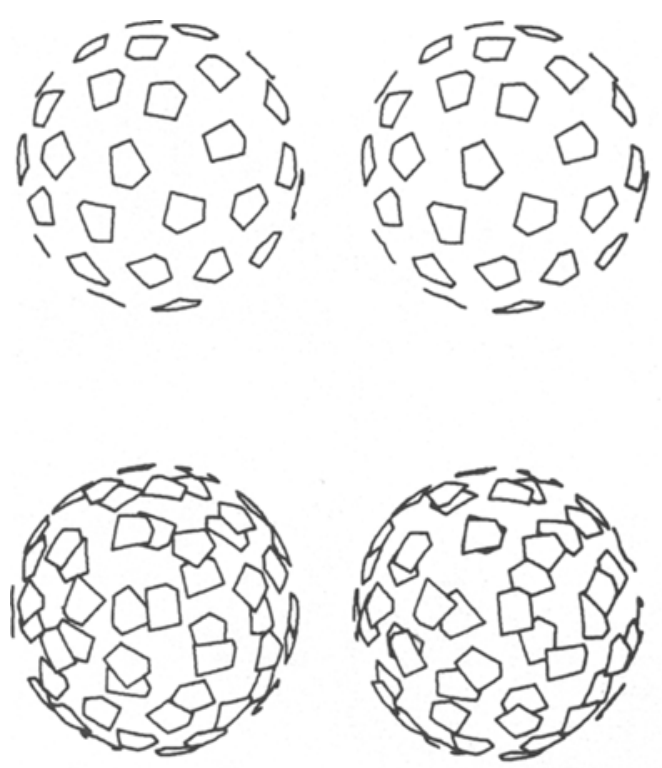

Figure 1. Frames from a dynamic stereoscopic display illustrating conflicting disparity and occlusion, for (a) edge occlusion and (b) element occlusion. (Film showed white figures on black.)
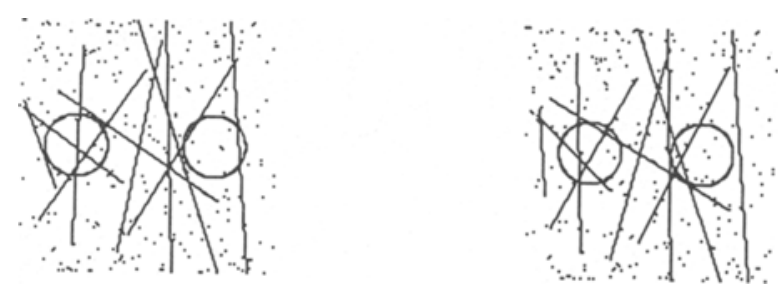

Figure 2. Sample frame from a dynamic kinetic depth display on a personal computer.

\section{Implications for Other Perceptual Judgments}

If kinetic depth and stereopsis are qualitatively different sources of information about three-dimensional relationships, can differences be found in their effects on other perceptual judgments? A particular judgment of interest is size constancy. When two figures are the same projected size, and disparity (or other depth information that specifies distance from the observer) indicates that they are at different distances, the object that is perceptually closer will usually appear smaller (although there are some paradoxical cases). Kinetic depth, on the other hand, does not specify near/far relationships. Depth order is ambiguous. Observers do report perceiving a particular depth order at a given time, however. In current research, we are addressing the question of whether the depth order perceived in an orthographic projection viewed monocularly affects perceived size. Casual observations of kinetic depth displays, and some preliminary studies, indicated that size constancy was absent or at least greatly reduced in these displays, relative to the levels found with stereoscopic viewing. With further refinements in the kinetic depth displays, however, we are beginning to find size constancy effects. Our current displays, shown in Figure 2, may seem to include an arbitrary assortment of features, but the selection of these features was based on four considerations developed in pilot studies: (1) Two "targets," the circles, were needed to allow the subject to make a ratio judgment of the perceived sizes. (2) A horizontal axis of rotation was selected so that the circles would be the same height in the image plane when the judgments were made, drawn across the cylinder at random positions to help prevent its perceptual collapse into two half cylinders. This sometimes occurs with rotating dot patterns, almost always with both halves of the pattern appearing convex. (4) The data were included to outline the cylinder, to facilitate judgments of depth relative to height.

\section{IMPLEMENTATION}

\section{Calculations}

Computer-generated dynamic stereoscopic displays are of course not new. Early research using random dot cinematograms is reviewed by Julesz (1971). The examples in this section do not include random dot backgrounds, but could be adapted to that method. 
The general method of computing coordinates for displays combining kinetic depth or motion parallax with stereoscopic depth is a simple modification of the method for producing dynamic monocular displays described by Braunstein (1976, Appendix A; see also Mingolla \& Todd, 1984). Consider one frame of a polar projection of points moving in three-dimensional space. Perspective effects are applied by multiplying the $X$ and $Y$ coordinate of each point by $E /(E-Z)$, where $E$ is the simulated viewing distance, $Z$ is the depth coordinate of the point and the projection plane is $Z=0$ (see Figure $3 a$ ). Horizontal disparity is applied by adding $\pm\left(d^{*} E\right) /(E-Z)$ to the $X$ coordinate, where $d$ is one half of the interocular distance (Figure 3b). A stereo display can be produced that is based on an orthographic projection of motion in depth, but there is a necessary inconsistency in the viewing distances simulated in the motion and in the stereo calculations. Orthographic projection implies infinite distance, which implies no disparity. This inconsistency is reflected in the computations when kinetic depth, with an orthographic projection, is combined with disparity. An orthographic projection can be produced by omitting the perspective multiplication described above, although it is sometimes more convenient to use a large $E$ value in the computation, effectively making the ratio of $E$ to $E-Z$ equal to 1.0. A smaller $E$ value, however, must be used in the disparity computation, simulating a close binocular viewing distance, if an effective stereoscopic display is to be produced. The use of two different $E$ values, one for computing motion and one for computing disparity, reflects the inconsistency between orthographic projection and disparity noted above.

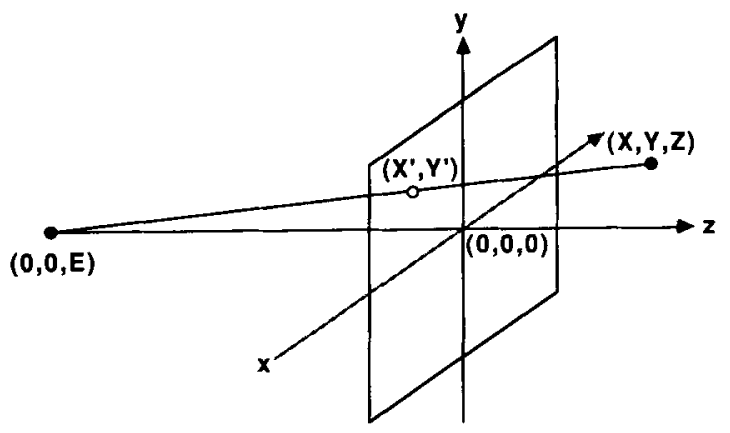

a

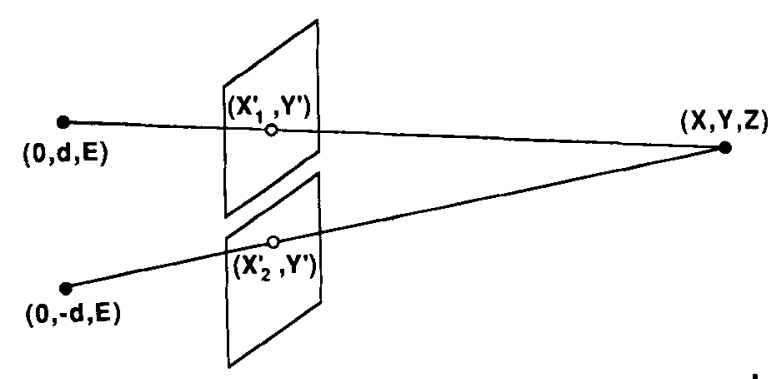

b

Figure 3. Projection of a point in three-dimensional space onto an image plane:(a) monocular and (b) stereo.
There is an alternative method of producing a dynamic stereo display if the rotation is about a vertical axis. Simply take two frames of the motion sequence, differing by about $5^{\circ}$ angular rotation, as the stereo pair (see Figure 1). This method does not overcome the inconsistency of combining disparity with an orthographic projection of motion, as the phase shift between the two views simulates convergence at a close viewing distance. It does have the practical advantages of not requiring separate generation of left and right frames. Stereo pairs based on rotation are currently used in dynamic stereo systems for medical imaging (Virapongse, Shapiro, Gmitro, \& Sarwar, 1986).

Stereo versions of translations along a horizontal axis similarly can be produced using frames from the motion sequence for the left and right images. In this case, the use of frames from the motion sequence is equivalent to the use of the horizontal disparity computations described above. This is because the incremental translation of a point in a perspective projection is proportional to its horizontal disparity in a stereo view. An orthographic projection of a horizontal translation would not be of interest, as all points would move at the same velocity in the image plane. Unlike the case of rotation about a vertical axis, succesive frames from an orthographic projection of a translation would not be useful as stereo pairs.

\section{Viewing Method}

Several methods of viewing dynamic stereo displays have appeared in the literature. The telestereoscope is an optical device for exaggerating disparity, and can be used for viewing a moving three-dimensional object (Epstein, 1968; Wallach et al., 1963). Shetty, Brodersen, and Fox (1979) developed a method that allows any moving (or nonmoving) object to be viewed as a red-green anaglyph. An electronic form generator and a random-dot generator are connected to the red and green amplifiers of a video monitor. Actual moving objects can be displayed by using two video cameras. The monitor is viewed with red/green glasses. This method can be adapted to crosspolarized viewing by using a videobeam projector and substituting polarizing filters for the red and green (or any two) color filters. Cross-polarized displays can also be presented by placing a liquid crystal polarizer in front of a monitor, with the alternation between horizontal and vertical polarization synchronized with the video display.

Another method of producing dynamic stereo displays uses two monitors to produce the images to be viewed by the two eyes (Gogel \& Tietz, 1976). Polarizing material is placed in front of each monitor and in front of the subject's eyes. The images are combined optically, for example, by placing the monitors at right angles and placing a half-silvered mirror at a $45^{\circ}$ angle to the monitors. Rogers and Collett (1986) used side-by-side monitors with a modified Wheatstone (mirror) stereoscope. In their motion parallax condition, the monitors were moved from side to side along a frontal parallel path.

We have been using a Brewster stereoscope in our current research. This is the familiar prism stereoscope used to view stereo drawings and photographs. A number of 
investigators have used similar approaches (e.g., Uttal, Fitzgerald, \& Eskin, 1975). Our choice of this method is based on its adaptability to a variety of displaygenerating systems, including motion pictures, pointplotting scopes, and personal computer displays. This adaptability arises because almost any system can be used to generate two images side by side on the same screen. There is a cost in resolution, however, as only a portion of the screen can be used for each member of the stereo pair. We have found it necessary to make one modification in a commercially available stereoscope (Stoetling Co., Chicago): To avoid undesirable reflections, we painted all surfaces flat black (after temporarily removing the prisms). Useful information about accommodation and convergence with the Brewster stereoscope can be found in an article by Eskridge (1976).

We have been using four display systems. For vector displays that cannot be drawn in real time, we have used stop frame animation, using a Tektronix 4010 storage scope with 1024 horizontal $\times 780$ vertical resolution and a 16-mm Automax camera. Resolutions of at least 420 $\times 420$ can be obtained for each member of the stereo pair. The film is projected onto a translucent screen and viewed from the opposite side through the stereoscope. For realtime, high-resolution dot displays, we have been using a Hewlett-Packard 1321B display scope, controlled by a PDP-11/44 computer using digital-to-analog channels. This provides an overall resolution of $4096 \times 4096$, and we have been able to obtain a resolution of $1800 \times 1800$ in each member of a stereo pair. Attenuators are used to reduce the image size for convenient viewing with the stereoscope. (The resolution figures for these two displays refer to the accuracy with which the dots or endpoints of vectors can be positioned.) These displays were used in Experiments 1 and 2, respectively, in Braunstein et al. (1986).

Convenient animation techniques are available with personal computers, although with lower resolution than the first two systems. We have been using an IBM-PC/AT with an AST Preview (Hercules-type) monochrome graphics board and a Princeton Max 12 amber monitor. (Many of the monochrome monitors available for personal computers have too long a phosphor persistence for acceptable animation. The Max 12 is adequate, but an even shorter persistence would be more desirable.) We have also been using an Apple Macintosh. These systems provide horizontal $\times$ vertical resolutions of $720 \times 350$ pixels and $650 \times 420$ pixels, respectively. With $512 \mathrm{~K}$ ram in each system, we can store just over 100 bit maps, with each bit map (one member of a stereo pair) $168 \times 126$ pixels on the AT and $125 \times 125$ pixels on the Macintosh. We are using Pascal on both systems. A programming example for the display in Figure 2, using Borland's Turbo Pascal and Turbo Graphix Toolbox on the IBM, is given in the Appendix. Julesz (personal communication, April 1986) has been producing dynamic random dot stereograms on the Apple Macintosh using the C language.

\section{CONCLUSION}

There are important research issues that require the combined study of different sources of information for the recovery of three-dimensional relationships from twodimensional images. The mutual constraints on threedimensional solutions provided by information from different sources and the specialization of sources for different types of depth information are examples of problems currently being addressed, both through computational analyses and empirical research. Both the study of stereoscopic depth perception and that of kinetic depth perception have been technically difficult in the past, but the combination of these sources is now easily accomplished on widely used personal computers without the need for special-purpose hardware or programming languages. This makes the study of these variables accessible to a larger number of researchers, including students at all levels. This should continue to move the information studied in research on depth perception closer to the complex combinations of information from multiple sources characteristic of a realistic three-dimensional environment.

\section{REFERENCES}

Andersen, G. J. (1986). The perception of self-motion: Psychophysical and computational approaches. Psychological Bulletin, 99, 52-65.

Andersen, G. J., \& Braunstein, M. L. (1983). Dynamic occlusion in the perception of rotation in depth. Perception \& Psychophysics, 34, 356-362.

Braunstein, M. L. (1966). Sensitivity of the observer to transformations of the visual field. Journal of Experimental Psychology, 72 , 683-687.

Braunstein, M. L. (1976). Depth perception through motion. New York: Academic Press.

Braunstein, M. L. (in press). The empirical study of structure from motion. In W. N. Martin \& J. K. Aggarwal (Eds.), Motion understanding: Robot and human vision. Hingham, MA: Kluwer Academic Publishers.

Braunstein, M. L., \& Andersen, G. J. (1981). Velocity gradients and relative depth perception. Perception \& Psychophysics, 29, 145-155.

Braunstein, M. L., Andersen, G. J., \& Riefer, D. M. (1982). The use of occlusion to resolve ambiguity in parallel projections. Perception \& Psychophysics, 31, 261-267.

Braunstein, M. L., Andersen, G. J., Rouse, M. W., \& Tittle, J. S. (1986). Recovering viewer-centered depth from disparity, occlusion, and velocity gradients. Perception \& Psychophysics, 40, 216-224.

Epstein, W. (1968). Modification of the disparity-depth relationship as a result of exposure to conflicting cues. American Journal of Psychology, 81, 189-197.

ESKRIDGE, J. B. (1976). Accommodation and vergence with the Brewster stereoscope. Journal of the American Optometric Association, 47, 919-924

Gogel, W C., \& Tietz, J. D. (1976). Adjacency and attention as determiners of perceived motion. Vision Research, 16, 839-845.

GREEN, B. F., JR. (1961). Figure coherence in the kinetic depth effect. Journal of Experimental Psychology, 62, 272-282.

JuLEsz, B. (1960). Binocular depth perception of computer-generated patterns. Bell System Technical Journal, 39, 1125-1162.

JULEsz, B. (1971). Foundations of cyclopean perception. Chicago: University of Chicago Press.

Koenderink, J. J (1986). Optic flow. Vision Research, 26, 161-180.

LAPPIN, J. S., \& LoVE, S. R. (1986). Stereoscopic structure from motion. Investigative Ophthalmology \& Visual Science, 27(3, Suppl.), 346. 
LURIa, S. M., \& WeIsSman, S. (1968). Relationship between static and dynamic stereo acuity. Joumal of Experimental Psychology, 76, 51-56.

MARR, D. (1982). Vision. San Francisco: Freeman.

Mingolla, E., \& Todd, J. T. (1984). Computational techniques for the graphic simulation of quadric surfaces. Journal of Experimental Psychology: Human Perception \& Performance, 10, 740-744.

Prazdny, K. (1986). Visual detection of three-dimensional structure from image movements without relevant luminance-domain motion signals. Manuscript submitted for publication.

REGAN, D., \& BEVERLEY, K. I. (1979). Binocular and monocular stimuli for motion in depth: Changing-disparity and changing-size feed the same motion-in-depth stage. Vision Research, 19, 1331-1342.

RicharDs, W. (1985). Structure from stereo and motion. Journal of the Optical Society of America, A2, 343-349.

Rogers, B. J. (1986). The perception of surface curvature from disparity and motion parallax cues. Investigative Ophthalmology \& Visual Science, 27(3, Suppl.), 181.

ROGERS, B. J., \& ColleTt, T. S. (1986). The appearance of surfaces specified by motion parallax and binocular disparity. Manuscript submitted for publication.

Rogers, B., \& Graham, M. (1979). Motion parallax as an independent cue for depth perception. Perception, 8, 125-134.
Schwartz, B. J. , \& SPERLING, G. (1983). Nonrigid 3D percepts from 2D representations of rigid objects. Investigative Ophthalmology \& Visual Science, 24(3, Suppl.), 239.

Shetty, S. S., BroderseN, A. J., \& Fox, R. (1979). System for generating dynamic random-element stereograms. Behavior Research Methods \& Instrumentation, 11, 485-490.

Ullman, S. (1979). The interpretation of visual motion. Cambridge, MA: MIT Press.

UtTal, W. R., Fitzgerald, J., \& Eskin, T. E. (1975). Parameters of tachistoscopic stereopsis. Vision Research, 15, 705-712.

Virapongse, C., Shapiro, M., Gmitro, A., \& SarWar, M. (1986). Three-dimensional computed tomographic reformation of the spine, skull, and brain from axial images. Neurosurgery, 18, 53-58.

Wallach, H., Moore, M. E., \& Davidson, L. (1963). Modification of stereoscopic depth-perception. American Journal of Psychology, 76, 191-204.

WALLACH, H., \& O' ConNELl, D. N. (1953). The kinetic depth effect. Journal of Experimental Psychology, 45, 205-217.

Waxman, A. M., d Duncan. J. H. (1985). Binocular image flows: Steps toward stereo-motion fusion (Report CAR-TR-119). College Park, MD: University of Maryland, Center for Automation Research.

\section{Simple Program for Stereoscopic Kinetic Depth Display}

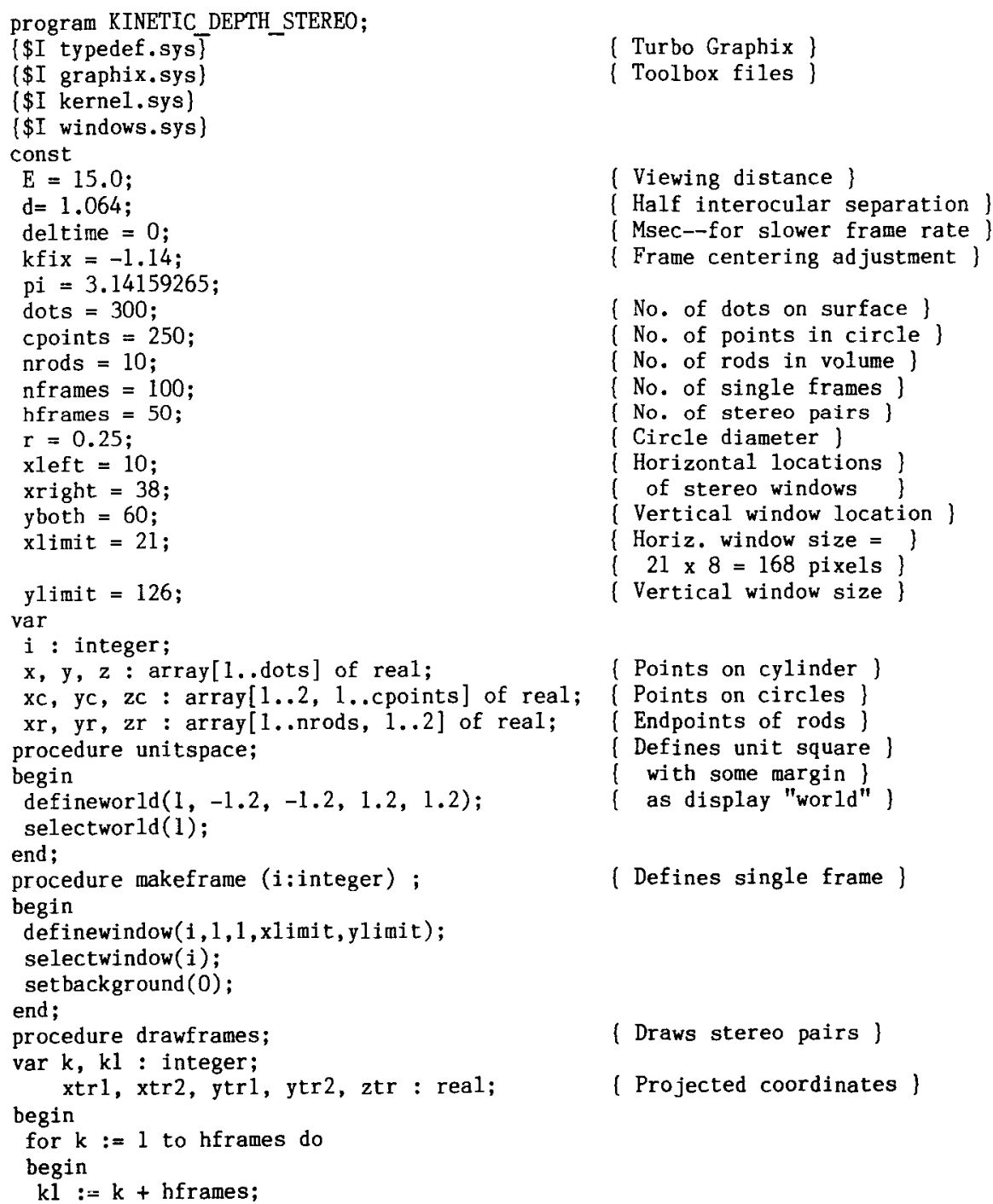

xtr1, xtr2, ytrl, ytr2, ztr : real; (Projected coordinates )

\{ Turbo Graphix \}

(Toolbox files \}

\{ Viewing distance \}

( Half interocular separation \}

(Msec--for slower frame rate)

\{Frame centering adjustment \}

(No. of dots on surface \}

(No. of points in circle

(No. of rods in volume \}

(No. of single frames \}

(No. of stereo pairs)

( Circle diameter )

(Horizontal locations )

of stereo windows

(Vertical window location

\{ Horiz. window size $=$ \}

$21 \times 8=168$ pixels

(Vertical window size)

\{ Points on cylinder \}

(Points on circles )

[ Endpoints of rods ]

\{Defines unit square \}

[ with some margin]

( as display "wor $1 d "$ )

\{ Defines single frame \}

(Draws stereo pairs) 


\section{APPENDIX (Continued)}

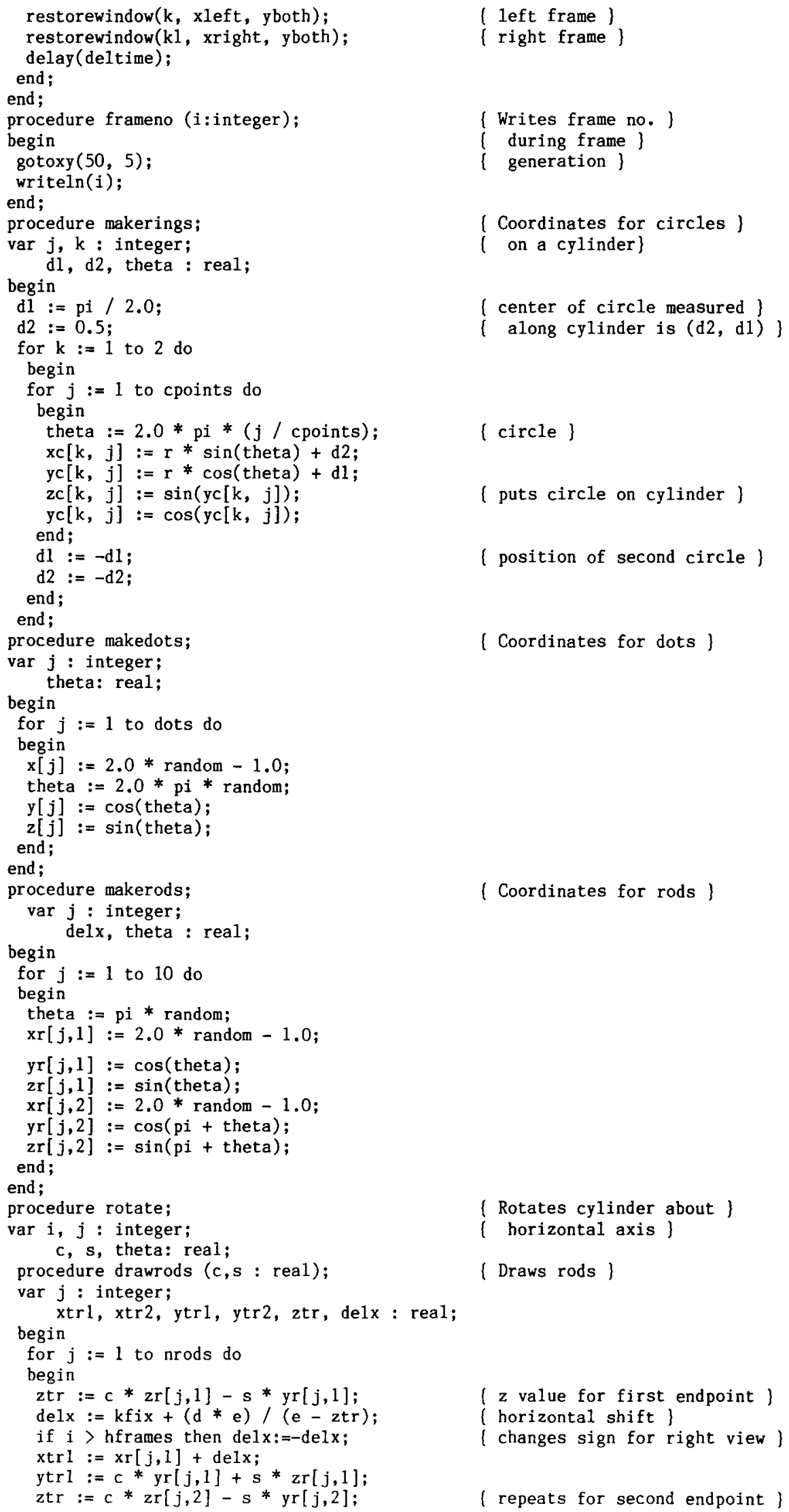

( left frame )

\{ right frame \}

$\left\{\begin{array}{c}\text { Writes frame no. }\} \\ \text { during frame }\}\end{array}\right.$

\{ generation \}

(Coordinates for circles )

\{ on a cylinder $\}$

( center of circle measured \}

( along cylinder is (d2, d1) \}

( circle)

\{ puts circle on cylinder \}

(position of second circle \}

\{ Coordinates for dots \}

( Coordinates for rods ) 


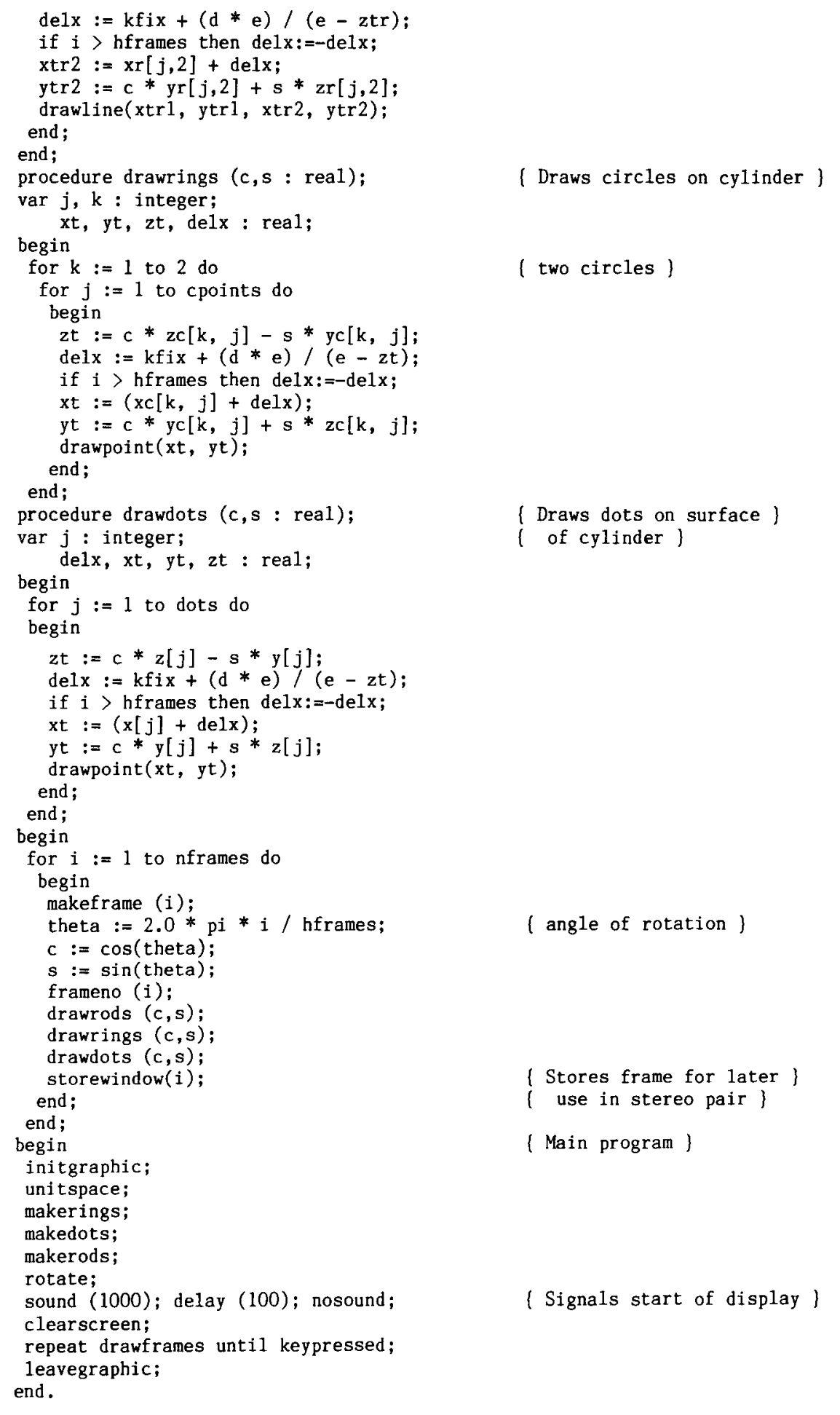

